\title{
Utilização, opinião e conhecimento dos profissionais de saúde sobre Equipamentos de Proteção Individual (EPIs) durante a pandemia de Sars-CoV-2: um estudo
}

\section{transversal}

\author{
Healthcare worker's use, opinion and knowledge of Personal Protective Equipment (PPE) during
}

the Sars-CoV-2 pandemic: a cross-sectional study

Uso, opinión y conocimientos de los profesionales de salud sobre los Equipos de Protección

Individual (EPI) durante la pandemia de Sars-CoV-2: un estudio transversal

Recebido: 30/08/2021 | Revisado: 06/09/2021 | Aceito: 09/09/2021 | Publicado: 12/09/2021

\author{
Thales Lima Lopes \\ ORCID: https://orcid.org/0000-0002-3262-0250 \\ Centro Universitário da Fundação Assis Gurgacz, Brasil \\ E-mail: thaleslimalopes@gmail.com \\ Andrey Felipe de Carvalho Pinheiro \\ ORCID: https://orcid.org/0000-0002-1404-2637 \\ Centro Universitário da Fundação Assis Gurgacz, Brasil \\ E-mail: andreyfelipe26@outlook.com \\ Cláudia Gregorini Silva \\ ORCID: https://orcid.org/0000-0001-5240-8567 \\ Centro Universitário da Fundação Assis Gurgacz, Brasil \\ E-mail: claudiagregorini@hotmail.com \\ Paula Carolina Mocelin \\ ORCID: https://orcid.org/0000-0002-2607-4062 \\ Centro Universitário da Fundação Assis Gurgacz, Brasil \\ E-mail: paula.cm@hotmail.com \\ Giovane Douglas Zanin \\ ORCID: https://orcid.org/0000-0003-2666-6081 \\ Centro Universitário da Fundação Assis Gurgacz, Brasil \\ E-mail: gdzanin@gmail.com
}

\begin{abstract}
Resumo
A prevenção e o controle das infecções ocupacionais requerem uma abordagem ampla com políticas bem-definidas e fundamentadas nas características das instituições de saúde. Nos trabalhadores da área de saúde, o risco ocupacional pode estar relacionado ao manuseio de materiais, produtos químicos ou equipamentos, que podem ser os causadores de acidentes de trabalho. O Equipamento de Proteção Individual (EPI) é uma das principais medidas de segurança a serem adotadas pelo profissional para garantir sua proteção. Dessa forma, o presente estudo teve como objetivo avaliar a relação do trabalhador de saúde com os EPIs e avaliar a quantidade de afastamentos por diagnóstico de infecção de vias aéreas superiores e consciência dos riscos que está exposto no trabalho. A maioria dos profissionais relatou uso de máscara $(55,93,2 \%)$, calçado fechado $(50,84,7 \%)$ e jaleco com manga longa $(44,74,6 \%)$. Contudo, houve menor adesão a utilização de luvas de procedimento (36, 61\%), toca ou capuz (32, 54,2\%) e óculos de proteção (19, 32,2\%). Embora cerca de um terço dos trabalhadores referirem utilizar óculos de proteção, quase a metade $(47,5 \%)$ acredita que este equipamento deveria obrigatoriamente ser utilizado durante a jornada de trabalho. 93,2\% dos entrevistados relataram utilizar EPIs por ter consciência dos riscos em que está exposto no trabalho. Com relação as análises inferenciais, não houve associação estatisticamente relevante entre afastamentos por infecções de vias aéreas superiores entre profissionais de saúde participantes do estudo com faixa etária $(p>0,05)$, sexo $(p>0,05)$, escolaridade ( $p>0,05)$, tipo de emprego ( $p>0,05)$, função $(p>0,05)$ e UBS de atuação ( $p>0,05)$. Dessa forma, pode-se concluir que é de grande importância para o aumento da segurança dos trabalhadores da saúde treinamentos para demonstrar aos profissionais a importância da utilização de EPIs e o modo correto de utilização destes equipamentos.
\end{abstract}

Palavras-chave: Equipamento de proteção individual; Profissionais da saúde; COVID-19.

\begin{abstract}
The prevention and control of occupational infections require a broad approach with well-defined policies based on the characteristics of health institutions. In healthcare workers, occupational risk may be related to the handling of materials, chemical products, or equipment, which can be the cause of work accidents. Personal Protective Equipment (PPE) is
\end{abstract}


one of the main safety measures to be adopted by professionals to ensure their protection. Thus, the present study aimed to evaluate the relationship between health workers and PPE and to assess the number of leaves of absence due to upper airway infection diagnosis and awareness of the risks they are exposed to at work. The vast majority of professionals reported using masks $(55,93.2 \%)$, closed shoes $(50,84.7 \%)$, and long-sleeved coat $(44,74.6 \%)$. However, there was less adherence to the use of procedure gloves $(36,61 \%)$, cap or hood $(32,54.2 \%)$, and eyewear $(19,32.2 \%)$. Although about one third of the workers reported using eyewear, almost half $(47.5 \%)$ believe that this equipment should be mandatory during the workday. $93.2 \%$ of the interviewees mentioned using PPE because they are aware of the risks they are exposed to at work. Regarding the inferential analyses, there was no statistically relevant association between days away from work due to upper airway infections among health professionals participating in the study with age $(p>0.05)$, gender $(p>0.05)$, education $(p>0.05)$, type of job $(p>0.05)$, function $(p>0.05)$ and UBS $(p>0.05)$. Thus, it can be concluded that it is of great importance to increase the safety of health workers through training to demonstrate to professionals the importance of using PPE and the correct way to use this equipment.

Keywords: Personal protective equipment; Health professionals; COVID-19.

\section{Resumen}

La prevención y el control de las infecciones profesionales requieren un enfoque amplio con políticas bien definidas basadas en las características de las instituciones de salud. En el personal sanitario, el riesgo laboral puede estar relacionado con la manipulación de materiales, productos químicos o equipos, que pueden ser la causa de accidentes laborales. Los equipos de protección individual (EPI) son una de las principales medidas de seguridad que deben adoptar los profesionales para garantizar su protección. Así pues, este estudio busca evaluar la relación entre los trabajadores sanitarios y los EPI y evaluar el número de días de ausencia del trabajo debido al diagnóstico de infección del tracto respiratorio superior y conciencia de los riesgos que están expuestos en el trabajo. La mayoría de los profesionales informaron el uso de mascarilla $(55,93,2 \%)$, zapatos cerrados $(50,84,7 \%)$ y bata de manga larga $(44,74,6 \%)$. Sin embargo, hubo una menor adherencia al uso de guantes de procedimiento $(36,61 \%)$, gorro o capucha $(32,54,2 \%)$ y anteojos de protección $(19,32,2 \%)$. Aunque cerca de un tercio de los trabajadores declararon utilizar anteojos de protección, casi la mitad $(47,5 \%)$ cree que este equipo debería ser obligatorio durante la jornada laboral. 93,2\% de los entrevistados afirma utilizar los EPI porque son conscientes de los riesgos a los que se exponen en el trabajo. En cuanto a los análisis inferenciales, no hubo asociación estadísticamente relevante entre las ausencias debidas a infecciones de las vías respiratorias superiores entre los profesionales de la salud que participaron en el estudio con el grupo de edad $(p>0,05)$, el género $(p>0,05)$, la educación $(p>0,05)$, el tipo de trabajo $(p>0,05)$, la función ( $p>0,05)$ y el UBS de trabajo $(\mathrm{p}>0,05)$. Por lo tanto, se puede concluir que es de gran importancia para aumentar la seguridad de los trabajadores de la salud capacitaciones para demostrar a los profesionales la importancia de usar el EPI y la forma correcta de utilizar este equipo.

Palabras clave: Equipo de protección personal; Profesionales de la salud; COVID-19.

\section{Introdução}

Os equipamentos de proteção individual são regulamentados pela Portaria 3.214 da Norma Regulamentadora NR-6 do Ministério do Trabalho. Tais equipamentos minimizam a possibilidade de contato direto dos trabalhadores com os agentes microbiológicos, o que assegura um trabalho eficaz e com menor incidência de ocorrer erros humanos do tipo não intencional (Carvalho \& Chaves, 2010).

A prevenção e o controle das infecções ocupacionais requerem uma abordagem ampla com políticas bem-definidas e fundamentadas nas características das instituições de saúde e na legislação em vigor, na medicina, higiene e a segurança do trabalho. Contudo, especialmente após o surgimento do Vírus da Imunodeficiência Humana (HIV), causador da AIDS, é que o contexto passou a ser evidenciado e preocupar-se com a biossegurança dos trabalhadores (Carvalho \& Chaves, 2010).

Nos profissionais da área de saúde, o risco ocupacional pode estar relacionado ao manuseio de materiais, produtos químicos ou equipamentos, que podem ser os causadores de acidentes de trabalho que irão refletir em sua vida pessoal e profissional (Silva, Sampaio \& Silva, 2016).

O Equipamento de Proteção Individual (EPI) é uma das principais medidas para a prevenção de acidentes de trabalho (Tribunal Superior do Trabalho, 2020). A Norma Regulamentadora 6 (NR-6) estabelece protocolos técnicos cientificos para a utilização dos EPIs durante a assistência ao paciente, sendo estes protocolos definidos no uso de máscara, óculos, avental/capote e lavagens das mãos para evitar a contaminação de quem presta a assistência bem como de quem recebe (contaminação cruzada e/ou disseminada) (Ministério do Trabalho, 2010; Chia et al, 2005). 
Já a Norma Regulamentadora 32 (NR-32) têm por finalidade estabelecer as diretrizes básicas para a implantação de medidas de proteção a segurança e a saúde dos trabalhadores dos serviços de saúde, bem como daqueles que exercem atividades de promoção a saúde em geral. A NR-32 classifica como serviços de saúde qualquer edificação destinada a prestação de assistência a saúde da população e todas as ações de promoção, recuperação, assistência, pesquisa e ensino em saúde em qualquer nível de complexidade. (Ministério do Trabalho, 2011).

Os Equipamentos de Proteção Coletiva (EPC) são aqueles que devem ser fornecidos pela empresa com o objetivo de proteger os trabalhadores dos riscos fornecidos pelo ambiente de trabalho, de maneira coletiva. Em outras palavras, são equipamentos que garantem a segurança do trabalho enquanto um grupo de pessoas (trabalhadores) executam uma determinada atividade ou tarefa. Eles têm como objetivo prevenir acidentes envolvendo trabalhadores ou qualquer terceiro que esteja transitando pelo ambiente, reduzir ou até mesmo anular qualquer risco comum à todos os colaboradores que o ambiente de trabalho possa fornecer e, por fim, minimizar perdas e aumentar a produtividade, ao fornecer aos trabalhadores um local de trabalho mais seguro. Os EPCs são instalados nos postos de trabalho, podendo ser fixos ou móveis e, diferentemente dos EPI's, fornecem proteção à mais de um trabalhador ao mesmo tempo (Sousa, Sousa \& Oliveira, 2019; Silva et al, 2018; Martins et al, 2014).

Diversos estudos associaram a utilização de EPIs com proteção ao desenvolvimento de infecções do trato respiratório em profissionais da saúde (Offeddu et al, 2016; MacIntyre et al, 2014; Ma et al, 2004). Em um contexto de pandemia profissionais de saúde devem utilizar os equipamentos adequados e cumprir as normas de biossegurança estabelecidas (Hossain et al, 2021; Furman et al, 2021).

Dessa forma, o presente estudo teve como objetivo avaliar a relação do trabalhador de saúde com os EPIs. Além disso, foram realizadas associações bivariadas entre quantidade de afastamentos por diagnóstico de infecção de vias aéreas superiores e consciência dos riscos que está exposto no trabalho com faixa etária, sexo, escolaridade, tipo de emprego e função do trabalhador de saúde.

\section{Metodologia}

Trata-se de um estudo quantitativo e descritivo. A amostra constituiu-se de trabalhadores da saúde atuantes na atenção primária de um município do Oeste do Paraná, que responderam individualmente um questionário com 16 perguntas, produzido pelos pesquisadores, abordando temas referentes a quais Equipamentos de Proteção Individual (EPIs) o profissional utiliza, quais julga que deveriam obrigatoriamente ser utilizados, dentre outros. O estudo foi aprovado pelo Comitê de Ética em Pesquisa do Centro Universitário da Fundação Assis Gurgacz com número CAAE 45644021.3.0000.5219. Como base metodológica utilizouse o trabalho de Pereira et al (2018).

Os dados obtidos foram alocados, tabulados e processados eletronicamente utilizando o programa Microsoft Office Excel e RStudio. Os dados quantitativos foram apresentados com base nas frequências relativas e, a seguir, se procedeu à realização da análise bivariada utilizando o teste de Fisher com nível de significância escolhido de $5 \%(\alpha=0,05)$. Nos casos em que uma das categorias apresentava frequência igual a 0 procedeu-se a utilização da correção de Haldane-Anscombe para cálculo da razão de chances e valor de p (Anscombe, 1956; Haldane, 1956; Hess \& Hess, 2017; Kim, 2017).

\section{Resultados e Discussão}

A Tabela 1 demonstra as características dos participantes da pesquisa. Foram analisados questionários de trabalhadores de 7 unidades de saúde, sendo as mais representativas a da Vila Tolentino (27,1\%), do Maria Luiza (22\%) e Pacaembu (18,6\%). A maioria dos entrevistados apresenta idade acima de 40 anos (57,6\%), é do sexo feminino (83,1\%), tem ensino superior (64,4\%). As profissões com mais participantes foram técnico em enfermagem $(18,6 \%)$, enfermeiro $(13,6 \%)$ e médicos $(13,6 \%)$. A 
principal forma de contratação dos profissionais é via estatutária $(83,1 \%)$ e para $62,7 \%$ o emprego público é a única fonte de renda.

Essas informações são condizentes com dados da literatura, que demonstram que a maioria dos trabalhadores dos setores de saúde são do sexo feminino (Tomasi et al, 2008; Fonseca et al, 2014; Souza et al, 2010) e o emprego público é o único trabalho destes profissionais (Tomasi et al, 2008; Fonseca et al, 2014), especialmente agentes Comunitários da Saúde (Fonseca et al, 2014).

Tabela 1: Descrição dos participantes do estudo por faixa etária, sexo, escolaridade, função, forma de contratação, tipo de contratação, tipo de emprego e Unidade de Saúde onde atua (n=59).

\begin{tabular}{|c|c|c|}
\hline Característica & $\mathbf{n}$ & Frequência relativa \\
\hline \multicolumn{3}{|l|}{ Faixa etária } \\
\hline 18 a 29 anos & 8 & $13,6 \%$ \\
\hline 30 a 39 anos & 17 & $28,8 \%$ \\
\hline 40 a 49 anos & 22 & $37,3 \%$ \\
\hline 50 anos ou mais & 12 & $20,3 \%$ \\
\hline \multicolumn{3}{|l|}{ Sexo } \\
\hline Feminino & 49 & $83,1 \%$ \\
\hline Masculino & 9 & $15,3 \%$ \\
\hline Não informado & 1 & $1,7 \%$ \\
\hline \multicolumn{3}{|l|}{ Escolaridade } \\
\hline Ensino Profissionalizante & 3 & $5,1 \%$ \\
\hline Curso Técnico & 17 & $28,8 \%$ \\
\hline Ensino Superior & 38 & $64,4 \%$ \\
\hline Ensino Médio & 1 & $1,7 \%$ \\
\hline \multicolumn{3}{|l|}{ Função } \\
\hline $\overrightarrow{A d m i n i s t r a c ̧ a ̃ o}$ & 5 & $8,5 \%$ \\
\hline Assistente social & 6 & $10,2 \%$ \\
\hline Agente Comunitário de Saúde & 6 & $10,2 \%$ \\
\hline Agente de Controle de Endemias & 1 & $1,7 \%$ \\
\hline Atendente & 2 & $3,4 \%$ \\
\hline Dentista & 4 & $6,8 \%$ \\
\hline Enfermeiro & 8 & $13,6 \%$ \\
\hline Médico & 8 & $13,6 \%$ \\
\hline Outros & 8 & $13,6 \%$ \\
\hline Técnico de enfermagem & 11 & $18,6 \%$ \\
\hline \multicolumn{3}{|l|}{ Forma de contratação } \\
\hline CLT & 1 & $1,7 \%$ \\
\hline Estatutário & 49 & $83,1 \%$ \\
\hline Outro & 9 & $15,3 \%$ \\
\hline \multicolumn{3}{|l|}{ Tipo de emprego } \\
\hline Único local de trabalho & 37 & $62,7 \%$ \\
\hline $\begin{array}{l}\text { Tem emprego em outro serviço de } \\
\text { saúde }\end{array}$ & 12 & $20,3 \%$ \\
\hline $\begin{array}{l}\text { Tem outra atividade remunerada ou } \\
\text { fonte de renda fora da área da saúde }\end{array}$ & 9 & $15,3 \%$ \\
\hline Não informado & 1 & $1,7 \%$ \\
\hline \multicolumn{3}{|l|}{ Unidade de Saúde } \\
\hline Maria Luiza & 13 & $22 \%$ \\
\hline Pacaembu & 11 & $18,6 \%$ \\
\hline Pioneiros & 7 & $11,9 \%$ \\
\hline Presidente & 10 & $16,9 \%$ \\
\hline Neva & 1 & $1,7 \%$ \\
\hline Vila Tolentino & 16 & $27,1 \%$ \\
\hline XIV de novembro & 1 & $1,7 \%$ \\
\hline
\end{tabular}


Com relação a utilização de Equipamentos de Proteção Individual (EPIs) pelos profissionais de saúde, a grande maioria dos profissionais relatou uso de máscara $(93,2 \%)$, calçado fechado $(84,7 \%)$ e jaleco com manga longa $(74,6 \%)$. Contudo, houve menor adesão a utilização de luvas de procedimento (61\%), toca ou capuz $(54,2 \%)$ e óculos de proteção $(32,2 \%)$ (Tabela 2).

A utilização de EPIs como luvas de procedimento pelos profissionais de saúde deve ser incentivada pois reduz as chances de contaminação bacteriana (Picheansanthian \& Chotibang, 2015). No caso dos óculos de proteção, acredita-se que pode reduzir o risco de infecções virais (Mermel, 2018; Wang \& Deng, 2020; Chu et al, 2020). Dessa forma, embora cerca de um terço $(32,2 \%)$ dos trabalhadores referem utilizar óculos de proteção, quase a metade $(47,5 \%)$ acredita que este equipamento deveria obrigatoriamente ser utilizado durante a jornada de trabalho (Tabela 2). A baixa utilização de óculos de proteção também é relatada em outros trabalhos (Rezende et al, 2012)

Quando questionados sobre motivos que levam a utilizar EPIs, 93,2\% dos entrevistados referiram utilizá-los por ter consciência dos riscos em que está exposto no trabalho (Tabela 2). Deve-se levar em conta que este estudo foi realizado durante a pandemia de Sars-CoV-2, o que possivelmente aumentou a consciência dos trabalhadores sobre a importância destes equipamentos.

Referente a presença de Equipamentos de Proteção Coletiva, 91,5\% dos participantes do estudo referiram presença de caixa para descarte de perfurocortantes em seu local de trabalho. Considerando que pelo menos um profissional de cada unidade de saúde referiu a presença deste objeto pode-se inferir que alguns trabalhadores não sabem que tal equipamento está disponível para utilização no local de trabalho.

Apenas 28,8\% dos participantes do estudo relataram que não tiveram nenhum afastamento por diagnóstico de influenza (gripe), SARS-Cov-2 ou Infecção de Vias Aéreas Superiores no período entre 2019 e 2020 (Tabela 2). Como os profissionais de saúde estão constantemente expostos a patógenos infectantes é essencial o uso de equipamentos de proteção, como demonstrado por estudos que associam menor quantidade de infecções respiratórias em profissionais da saúde que utilizam EPIs adequadamente (Suzuki et al, 2021; Chu et al, 2020; Offeddu et al, 2016).

Apenas 72,9\% dos profissionais entrevistados acreditam que o uso dos EPIs e EPCs é feito da maneira correta (Tabela 2). Medidas como treinamento podem ser consideradas para ensinar aos profissionais a forma ótima de utilização destes equipamentos (Doll et al, 2017; Barratt, Shaban \& Gilbert, 2020). 
Tabela 2: Descrição dos participantes referente ao uso de Equipamentos de Proteção Individual (EPIs), motivos para utilizá-los, presença de Equipamentos de Proteção Coletiva (EPCs) na unidade de saúde, quantas vezes se afastou do trabalho entre 2019-2020 devido a infecção respiratória, opinião referente a quais EPIs deveriam obrigatoriamente ser utilizados durante a jornada de trabalho e opinião sobre a utilização correta de EPIs e EPCs $(n=59)$.

\begin{tabular}{lcc}
\hline Característica & n & Frequência relativa \\
\hline EPIs que utiliza & & \\
Jaleco com manga longa & 44 & $74,6 \%$ \\
Máscara & 55 & $93,2 \%$ \\
Óculos de proteção & 19 & $32,2 \%$ \\
Toca ou capuz & 32 & $54,2 \%$ \\
Calçado fechado & 50 & $84,7 \%$ \\
Luvas de procedimento & 36 & $61 \%$
\end{tabular}

EPIs que acredita que deveriam obrigatoriamente ser utilizados durante a jornada de trabalho

$\begin{array}{lcc}\text { Jaleco com manga longa } & 50 & 84,7 \% \\ \text { Máscara } & 56 & 94,9 \% \\ \text { Óculos de proteção } & 28 & 47,5 \% \\ \text { Toca ou capuz } & 29 & 49,2 \% \\ \begin{array}{l}\text { Calçado fechado } \\ \text { Luvas de procedimento }\end{array} & 55 & 93,2 \% \\ \text { Motivos para usar EPIs } & 36 & 61 \% \\ \begin{array}{l}\text { Porque o empregador manda } \\ \text { Porque a lei ou norma }\end{array} & & 5,1 \% \\ \text { determina } & 3 & 6,8 \% \\ \begin{array}{l}\text { Porque tenho consciência dos } \\ \text { riscos que estou exposto no } \\ \text { trabalho }\end{array} & 4 & \\ \text { Não informado } & & 93,2 \% \\ \text { Caixa perfurocortante } & 55 & 3,4 \% \\ \text { Autoclave } & & \\ \text { Lava olhos } & 2 & 91,5 \% \\ \text { Câmara de fluxo laminar } & & 18,6 \% \\ \text { Q } & 54 & 0 \% \\ \end{array}$

Quantas vezes se afastou do trabalho entre 2019-2020 com diagnóstico de influenza (gripe), SARS-Cov-2 ou Infecção de Vias Aéreas Superiores?

\begin{tabular}{lcc}
0 & 17 & $28,8 \%$ \\
1 & 23 & $39 \%$ \\
2 & 14 & $23,7 \%$ \\
3 & 1 & $1,7 \%$ \\
4 ou mais & 3 & $5,1 \%$ \\
Não informado & 1 & $1,7 \%$ \\
credita que o uso de EPIs e EPCs é feito de maneira correta & \\
Não & 16 & $27,1 \%$ \\
Sim & 43 & $72,9 \%$ \\
\hline
\end{tabular}

Fonte: Autores (2021).

Na Tabela 3 foi descrito o conhecimento dos profissionais de saúde referente a questões jurídicas sobre os EPIs. Observou-se que em todas as questões o índice de acerto foi elevado, sendo apenas a questão referente a possibilidade de punição com medidas disciplinares em profissionais que não utilizam EPIs com mais de 10\% de respostas incorretas (10,2\%). Portanto, é importante informar aos trabalhadores que responderam equivocadamente que nos casos onde EPIs não são utilizados existe a possibilidade de medidas disciplinares. 
Tabela 3: Respostas dos trabalhadores atuantes na atenção primária do município de Cascavel/PR referente a utilização de Equipamentos de Proteção Individual (EPI) (n=59).

\begin{tabular}{|c|c|c|}
\hline Afirmativa & $\begin{array}{l}\text { Responderam } \\
\text { corretamente }\end{array}$ & $\%$ \\
\hline $\begin{array}{l}\text { O fornecimento do EPI é dever do } \\
\text { empregador e o seu custo pode ser } \\
\text { cobrado do trabalhador - Falso }\end{array}$ & (2) & $96,6 \%$ \\
\hline $\begin{array}{l}\text { O fornecimento do EPI é dever do } \\
\text { empregador e o seu custo NÃO pode } \\
\text { ser cobrado do trabalhador - } \\
\text { Verdadeiro }\end{array}$ & 58 & $98,3 \%$ \\
\hline $\begin{array}{l}O \text { fornecimento do EPI é de } \\
\text { responsabilidade do trabalhador - } \\
\text { Falso }\end{array}$ & 55 & $93,2 \%$ \\
\hline $\begin{array}{l}\text { Uma vez recebido o EPI o } \\
\text { trabalhador não tem obrigações } \\
\text { para a conservação do mesmo - } \\
\text { Falso }\end{array}$ & 57 & $96,6 \%$ \\
\hline $\begin{array}{l}\text { O uso do EPI é uma opção do } \\
\text { trabalhador, ou seja, ele usa o que } \\
\text { quiser e se quiser - Falso }\end{array}$ & 57 & $96,6 \%$ \\
\hline $\begin{array}{l}\text { A falta de uso do EPI pode ser } \\
\text { punida com medidas disciplinares - } \\
\text { Verdadeiro }\end{array}$ & 53 & $89,8 \%$ \\
\hline $\begin{array}{l}\text { O dever é mútuo, pois o empregador } \\
\text { deve fornece-los e o trabalhador } \\
\text { deve usá-los - Verdadeiro }\end{array}$ & 58 & $98,3 \%$ \\
\hline
\end{tabular}

Fonte: Autores (2021).

Não houve associação estatisticamente relevante entre acreditar que uso de EPIs e EPCs é realizado de forma correta comparando os profissionais com 50 anos ou mais com trabalhadores na faixa etária entre 18 a 29 anos (p=0,37), 30 a 39 anos ( $\mathrm{p}=0,24)$ e 40 a 49 anos ( $\mathrm{p}=0,11)$. Também não foi demonstrado associação estatisticamente relevante envolvendo sexo ( $\mathrm{p}=0,24)$, escolaridade $(p>0,05)$ e tipo de emprego $(p>0,05)$. Apenas os agentes comunitários de saúde acreditam menos que uso de EPIs e EPCs é feito de maneira correta na comparação com profissionais técnicos em enfermagem $(\mathrm{p}<0,01)($ Tabela 4). 
Tabela 4: Associação bivariada entre acreditar que uso de Equipamentos de Proteção Individual (EPI) e Equipamentos de Proteção Coletiva (EPC) é feito da maneira correta com faixa etária, sexo, escolaridade, tipo de emprego e função.

\begin{tabular}{|c|c|c|c|c|}
\hline $\begin{array}{l}\text { Acredita que uso de EPIs } \\
\text { e EPCs é feito da maneira } \\
\text { correta? }\end{array}$ & $\operatorname{Sim}(\%)$ & Não (\%) & $\begin{array}{l}\text { Razão de } \\
\text { Chances } \\
\text { (IC 95\%) } \\
\end{array}$ & Valor de $p$ \\
\hline \multicolumn{5}{|l|}{ Faixa etária } \\
\hline 18 a 29 anos & $6(75 \%)$ & $2(25 \%)$ & $\begin{array}{c}3,00 \\
(0,42-21,30\end{array}$ & 0,37 \\
\hline 30 a 39 anos & $13(76,5 \%)$ & $4(23,5 \%)$ & $\begin{array}{c}3,25 \\
(0,66-15,98)\end{array}$ & 0,24 \\
\hline 40 a 49 anos & $18(81,8 \%)$ & $4(18,2 \%)$ & $\begin{array}{c}4,50 \\
(0,94-21,56)\end{array}$ & 0,11 \\
\hline 50 anos ou mais & $6(50 \%)$ & $6(50 \%)$ & 1 & \\
\hline \multicolumn{5}{|l|}{ Sexo } \\
\hline Feminino & $37(75,5 \%)$ & $12(24,5 \%)$ & $\begin{array}{c}2,47 \\
(0,57-10,70)\end{array}$ & 0,24 \\
\hline Masculino & $5(55,6 \%)$ & $4(44,4 \%)$ & 1 & \\
\hline \multicolumn{5}{|l|}{ Escolaridade } \\
\hline $\begin{array}{l}\text { Ensino } \\
\text { Profissionalizante }\end{array}$ & $1(33,3 \%)$ & $2(66,7 \%)$ & $\begin{array}{c}0,20 \\
(0,02-2,48)\end{array}$ & 0,23 \\
\hline Curso Técnico & $15(88,2 \%)$ & $2(11,8 \%)$ & $\begin{array}{c}3,06 \\
(0,60-15,65\end{array}$ & 0,30 \\
\hline Ensino Superior & $27(71,1 \%)$ & $11(28,9 \%)$ & 1 & \\
\hline \multicolumn{5}{|l|}{ Tipo de emprego } \\
\hline Único local de trabalho & $25(67,6 \%)$ & $12(32,4 \%)$ & 1 & \\
\hline $\begin{array}{l}\text { Tem emprego em outro } \\
\text { serviço de saúde }\end{array}$ & $11(91,7 \%)$ & $1(8,3 \%)$ & $\begin{array}{c}5,28 \\
(0,61-45,77\end{array}$ & 0,14 \\
\hline $\begin{array}{l}\text { Tem outra atividade } \\
\text { remunerada ou fonte de } \\
\text { renda fora da área da } \\
\text { saúde }\end{array}$ & $7(77,8 \%)$ & $2(22,2 \%)$ & $\begin{array}{c}1,68 \\
(0,30-9,34)\end{array}$ & 0,70 \\
\hline \multicolumn{5}{|l|}{ Função } \\
\hline Administração & $5(100 \%)$ & $0(0 \%)$ & $\begin{array}{c}1,57 \\
(0,05-45,37) *\end{array}$ & $0,53 *$ \\
\hline Assistente social & $4(66,7 \%)$ & $2(33,3 \%)$ & $\begin{array}{c}0,20 \\
(0,01-2,88)\end{array}$ & 0,51 \\
\hline $\begin{array}{l}\text { Agente Comunitário de } \\
\text { Saúde }\end{array}$ & $1(16,7 \%)$ & $5(83,3 \%)$ & $\begin{array}{c}0,02 \\
(0,00-0,39)\end{array}$ & $<0,01$ \\
\hline Enfermeiro & $7(87,5 \%)$ & $1(12,5 \%)$ & $\begin{array}{c}0,70 \\
(0,04-13,18)\end{array}$ & 1 \\
\hline Médico & $5(62,5 \%)$ & $3(37,5 \%)$ & $\begin{array}{c}0,17 \\
(0,01-2,04\end{array}$ & 0,26 \\
\hline $\begin{array}{l}\text { Técnico } \\
\text { enfermagem }\end{array}$ & $10(90,9 \%)$ & $1(9,1 \%)$ & 1 & \\
\hline
\end{tabular}

* Correção de Haldane-Anscombe. Fonte: Autores (2021).

A Tabela 5 demonstra a associação bivariada entre afastamentos do trabalho por diagnóstico de influenza (gripe), SARSCoV-2 ou Infecção de Vias Aéreas Superiores. Não houve associação estatisticamente relevante entre afastamentos por esta causa com faixa etária ( $p>0,05)$, sexo $(p>0,05)$, escolaridade $(p>0,05)$, tipo de emprego ( $>00,05)$, função ( $p>0,05)$ e UBS de atuação ( $p>0,05)$. Destaca-se que apenas 53,8\% dos profissionais atuantes na Unidade de Saúde Maria Luiza tiveram este tipo de afastamento, na comparação com 81,8\% dos trabalhadores da UBS Pacaembu, 71,4\% da unidade localizada no bairro Pioneiros, $80 \%$ da UBS Presidente e $81,2 \%$ do posto de saúde do bairro Vila Tolentino. Contudo, essa diferença não é estatisticamente relevante $(p>0,05)$. 
Na Tabela 6 foi analisado o uso de EPIs por ter consciência dos riscos que o profissional está exposto no trabalho. Não houve associação estatisticamente relevante entre uso dos EPIs por esta motivação pela faixa etária $(p>0,05)$, sexo $(p>0,05)$, escolaridade ( $p>0,05)$, tipo de emprego $(p>0,05)$ e função $(p>0,05)$.

Tabela 5: Associação bivariada entre afastamentos com diagnóstico de influenza (gripe), SARS-CoV-2 ou infecção de vias aéreas superiores com faixa etária, sexo, escolaridade, tipo de emprego, função e UBS onde atua.

\begin{tabular}{|c|c|c|c|c|}
\hline $\begin{array}{l}\text { Afastamentos com diagnóstico } \\
\text { de influenza (gripe), SARS-Cov- } \\
2 \text { ou Infecção de Vias Aéreas } \\
\text { Superiores? }\end{array}$ & $\begin{array}{c}\geq 1 \text { afastamentos } \\
(\%)\end{array}$ & $\begin{array}{c}0 \text { afastamentos } \\
(\%)\end{array}$ & $\begin{array}{l}\text { Razão de } \\
\text { Chances } \\
\text { (IC 95\%) }\end{array}$ & Valor de $p$ \\
\hline \multicolumn{5}{|l|}{ Faixa etária } \\
\hline 18 a 29 anos & $5(62,5 \%)$ & $3(37,5 \%)$ & 1 & \\
\hline 30 a 39 anos & $13(76,5 \%$ & $4(23,5 \%)$ & $\begin{array}{c}1,95 \\
(0,32-12,01)\end{array}$ & 0,64 \\
\hline 40 a 49 anos & $16(72,7 \%)$ & $6(27,3 \%)$ & $\begin{array}{c}1,60 \\
(0,29-8,86)\end{array}$ & 0,66 \\
\hline 50 anos ou mais & $8(66,7 \%)$ & $4(33,3 \%)$ & $\begin{array}{c}1,20 \\
(0,19-7,77)\end{array}$ & \\
\hline \multicolumn{5}{|l|}{ Sexo } \\
\hline Feminino & $34(69,4 \%)$ & $15(30,6 \%)$ & 1 & \\
\hline Masculino & $7(77,8 \%)$ & $2(22,2 \%)$ & $\begin{array}{c}1,54 \\
(0,29-8,33)\end{array}$ & 1 \\
\hline \multicolumn{5}{|l|}{ Escolaridade } \\
\hline Ensino Profissionalizante & $3(100 \%)$ & $0(0 \%)$ & $\begin{array}{c}3,30 \\
(0,16-68,93) *\end{array}$ & $0,31 *$ \\
\hline Curso Técnico & $13(76,5 \%)$ & $4(23,5 \%)$ & $\begin{array}{c}1,50 \\
(0,40-5,58)\end{array}$ & 0,75 \\
\hline $\begin{array}{l}\text { Ensino Superior } \\
\text { Tipo de emprego }\end{array}$ & $26(68,4 \%)$ & $12(31,6 \%)$ & 1 & \\
\hline Único local de trabalho & $27(73 \%)$ & $10(27 \%)$ & $\begin{array}{c}1,35 \\
(0,28-6,45)\end{array}$ & 0,70 \\
\hline $\begin{array}{l}\text { Tem emprego em outro } \\
\text { serviço de saúde }\end{array}$ & $9(75 \%)$ & $3(25 \%)$ & $\begin{array}{c}1,50 \\
(0,22-10,08)\end{array}$ & 1 \\
\hline $\begin{array}{l}\text { Tem outra atividade } \\
\text { remunerada ou fonte de renda } \\
\text { fora da área da saúde }\end{array}$ & $6(66,7 \%)$ & $3(33,3 \%)$ & 1 & \\
\hline \multicolumn{5}{|l|}{ Função } \\
\hline Administração & $4(80 \%)$ & $1(20 \%)$ & $\begin{array}{c}4,00 \\
(0,30-53,47)\end{array}$ & 0,56 \\
\hline Assistente social & $4(66,7 \%)$ & $2(33,3 \%)$ & $\begin{array}{c}2,00 \\
(0,22-17,89)\end{array}$ & 0,63 \\
\hline Agente Comunitário de Saúde & $5(83,3 \%)$ & $1(16,7 \%)$ & $\begin{array}{c}5,00 \\
(0,39-64,39)\end{array}$ & 0,30 \\
\hline $\begin{array}{l}\text { Enfermeiro } \\
\text { Médico }\end{array}$ & $\begin{array}{l}4(50 \%) \\
4(50 \%)\end{array}$ & $\begin{array}{l}4(50 \%) \\
4(50 \%)\end{array}$ & $\begin{array}{l}1 \\
1\end{array}$ & \\
\hline Técnico de enfermagem & $8(72,7 \%)$ & $3(27,3 \%)$ & $\begin{array}{c}2,67 \\
(0,39-18,17)\end{array}$ & 0,38 \\
\hline \multicolumn{5}{|l|}{ UBS de atuação } \\
\hline Pacaembu & $9(81,8 \%)$ & $2(18,2 \%)$ & $\begin{array}{c}3,86 \\
(0,59-25,29)\end{array}$ & 0,21 \\
\hline Pioneiros & $5(71,4 \%)$ & $2(28,6 \%)$ & $\begin{array}{c}2,14 \\
(0,30-15,35)\end{array}$ & 0,64 \\
\hline Presidente & $8(80 \%)$ & $2(20 \%)$ & $\begin{array}{c}3,43 \\
(0,52-22,80)\end{array}$ & 0,38 \\
\hline Vila Tolentino & $13(81,2 \%)$ & $3(18,8 \%)$ & $\begin{array}{c}3,71 \\
(0,70-19,59)\end{array}$ & 0,23 \\
\hline
\end{tabular}

* Correção de Haldane-Anscombe. Fonte: Autores (2021) 
Tabela 6: Associação bivariada entre uso de Equipamento de Proteção Individual (EPI) por ter consciência dos riscos que está exposto no trabalho com faixa etária, sexo, escolaridade, tipo de emprego e função.

\begin{tabular}{|c|c|c|c|c|}
\hline 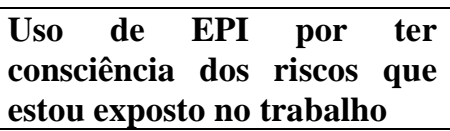 & $\operatorname{Sim}(\%)$ & Não (\%) & $\begin{array}{c}\text { Razão de } \\
\text { Chances } \\
\text { (IC 95\%) } \\
\end{array}$ & Valor de p \\
\hline \multicolumn{5}{|l|}{ Faixa etária } \\
\hline 18 a 29 anos & $7(87,5 \%)$ & $1(12,5 \%)$ & 1 & \\
\hline 30 a 39 anos & $16(94,1 \%)$ & $1(5,9 \%)$ & $\begin{array}{c}2,29 \\
(0,12-41,98)\end{array}$ & 1 \\
\hline 40 a 49 anos & $20(90,9 \%)$ & $2(9,1 \%)$ & $\begin{array}{c}1,43 \\
(0,11-18,30)\end{array}$ & 1 \\
\hline 50 anos ou mais & $12(100 \%)$ & $0(0 \%)$ & $\begin{array}{c}5,00 \\
(0,18-139,16) *\end{array}$ & $0,19 *$ \\
\hline \multicolumn{5}{|l|}{ Sexo } \\
\hline Feminino & $45(91,8 \%)$ & $4(8,2 \%)$ & 1 & \\
\hline Masculino & $9(100 \%)$ & $0(0 \%)$ & $\begin{array}{c}1,88 \\
(0,09-37,90) *\end{array}$ & $1 *$ \\
\hline \multicolumn{5}{|l|}{ Escolaridade } \\
\hline Ensino Profissionalizante & $2(66,7 \%)$ & $1(33,3 \%)$ & $\begin{array}{c}0,17 \\
(0,01-2,49)\end{array}$ & 0,27 \\
\hline Curso Técnico & $17(100 \%)$ & $0(0 \%)$ & $\begin{array}{c}3,45 \\
(0,17-70,57)\end{array}$ & 0,30 \\
\hline \multicolumn{5}{|l|}{ Tipo de emprego } \\
\hline Único local de trabalho & $34(91,9 \%)$ & $3(8,1 \%)$ & $\begin{array}{c}1,03 \\
(0,10-10,95)\end{array}$ & 1 \\
\hline $\begin{array}{l}\text { Tem emprego em outro } \\
\text { serviço de saúde }\end{array}$ & $11(91,7 \%)$ & $1(8,3 \%)$ & 1 & \\
\hline $\begin{array}{l}\text { Tem outra atividade } \\
\text { remunerada ou fonte de } \\
\text { renda fora da área da } \\
\text { saúde }\end{array}$ & $9(100 \%)$ & $0(0 \%)$ & $\begin{array}{c}2,48 \\
(0,09-68,14) *\end{array}$ & $0,49 *$ \\
\hline \multicolumn{5}{|l|}{ Função } \\
\hline Administração & $4(80 \%)$ & $1(20 \%)$ & $\begin{array}{c}2,00 \\
(0,13-31,98)\end{array}$ & 1 \\
\hline Assistente social & $4(66,7 \%)$ & $2(33,3 \%)$ & 1 & \\
\hline $\begin{array}{l}\text { Agente Comunitário de } \\
\text { Saúde }\end{array}$ & $6(100 \%)$ & $0(0 \%)$ & $\begin{array}{c}7,22 \\
(0,28-189,19) *\end{array}$ & $0,45 *$ \\
\hline Enfermeiro & $8(100 \%)$ & $0(0 \%)$ & $\begin{array}{c}9,44 \\
(0,37-242,18) *\end{array}$ & $0,16 *$ \\
\hline Médico & $8(100 \%)$ & $0(0 \%)$ & $\begin{array}{c}9,44 \\
(0,37-242,18) *\end{array}$ & $0,16 *$ \\
\hline Técnico de enfermagem & $11(100 \%)$ & $0(0 \%)$ & $\begin{array}{c}12,78 \\
(0,51-321,73) *\end{array}$ & $0,10 *$ \\
\hline
\end{tabular}

* Correção de Haldane-Anscombe. Fonte: Autores (2021).

\section{Conclusão}

Observou-se elevado percentual $(93,2 \%)$ de utilização de EPIs devido a consciência individual dos riscos que está exposto no trabalho (Tabela 2). Deve-se levar em conta que este estudo foi realizado durante a pandemia de Sars-CoV-2, o que possivelmente aumentou a consciência dos trabalhadores sobre a importância destes equipamentos.

Inferiu-se que quase $10 \%$ dos profissionais não sabem da presença de caixa para descarte de objetos perfurocortantes na unidade de saúde onde trabalham (Tabela 2). Como o descarte adequado desses materiais é de grande importância para a segurança dos trabalhadores da saúde medidas é fundamental que exista uma forma de comunicar a todos os profissionais a presença desse equipamento e a forma correta de realização do descarte. 
Deve ser avaliado pela Secretaria de Saúde os motivos que levaram aos agentes comunitários de saúde a acreditar menos que uso de EPIs e EPCs é feito de maneira correta na comparação com profissionais técnicos em enfermagem ( $<<0,01)($ Tabela 4). Uma das possibilidades para minimizar esta questão é ofertar aulas ensinando a forma correta de uso e manuseio destes equipamentos para os agentes comunitários de saúde terem mais segurança na utilização dos EPIs e EPCs. Além de focar nessa área onde foi localizado lacuna de conhecimento, para o aumento da segurança dos trabalhadores atuantes em estabelecimentos de saúde podem ser oferecidos cursos gerais de treinamento para demonstrar aos funcionários a importância da utilização de EPIs e o modo correto de utilização destes equipamentos.

Futuros estudos podem abordar o tema da relação dos trabalhadores de saúde com os EPIs e EPCs nas atençães secundárias e terciárias da saúde. Com os dados obtidos nesses trabalhos seria possível comparar as semelhanças e diferenças envolvendo a utilização destes equipamentos nos diversos graus de atenção e, dessa forma, ter subsídios para intervenções mais adequadas a cada tipo de estabelecimento de saúde para melhoria da segurança dos profissionais.

\section{Referências}

Anscombe, F. J. (1956). On Estimating Binomial Response Relations. Biometrika, 43(3/4), 461. https://doi.org/10.2307/2332926

Barratt, R., Shaban, R. Z., \& Gilbert, G. L. (2020). Characteristics of personal protective equipment training programs in Australia and New Zealand hospitals: A survey. Infection, Disease \& Health, 25(4), 253-261. https://doi.org/10.1016/j.idh.2020.05.005

Carvalho, J. F. de S., \& Chaves, L. D. P. (2010). Supervisão de enfermagem no uso de equipamento de proteção individual em um hospital geral. Cogitare Enfermagem, 15(3). https://doi.org/10.5380/ce.v15i3.18897

Chia, S. E. (2005). Appropriate use of personal protective equipment among healthcare workers in public sector hospitals and primary healthcare polyclinics during the SARS outbreak in Singapore. Occupational and Environmental Medicine, 62(7), 473-477. https://doi.org/10.1136/oem.2004.015024

Chu, D. K., Akl, E. A., Duda, S., Solo, K., Yaacoub, S., Schünemann, H. J., Chu, D. K., Akl, E. A., El-harakeh, A., Bognanni, A., Lotfi, T., Loeb, M., Hajizadeh, A., Bak, A., Izcovich, A., Cuello-Garcia, C. A., Chen, C., Harris, D. J., Borowiack, E., \& Schünemann, H. J. (2020). Physical distancing, face masks, and eye protection to prevent person-to-person transmission of SARS-CoV-2 and COVID-19: a systematic review and meta-analysis. The Lancet, 395(10242), 19731987. https://doi.org/10.1016/S0140-6736(20)31142-9

Doll, M., Feldman, M., Hartigan, S., Sanogo, K., Stevens, M., McReynolds, M., Masroor, N., Cooper, K., \& Bearman, G. (2017). Acceptability and Necessity of Training for Optimal Personal Protective Equipment Use. Infection Control\& Hospital Epidemiology, 38(2), 226-229. https://doi.org/10.1017/ice.2016.252

Fonseca, F. F., Lima, C. de A., Alves, J. P., Silva, S. S. de S., Lopes, M. C., Carneiro, J. A., \& Costa, F. M. da. (2014). Caracterização sociodemográfica e ocupacional de trabalhadores da Estratégia em Saúde da Família. Revista Eletrônica Gestão \& Saúde, 5, $2465-2478$.

Furman, E., Cressman, A., Shin, S., Kuznetsov, A., Razak, F., Verma, A., \& Diamant, A. (2021). Prediction of personal protective equipment use in hospitals during COVID-19. Health Care Management Science, 24(2), 439-453. https://doi.org/10.1007/s10729-021-09561-5

Haldane, J. B. S. (1956). The estimation and significance of the logarithm of a ratio of frequencies. Annals of Human Genetics, 20(4), 309-311. https://doi.org/10.1111/j.1469-1809.1955.tb01285.x

Hess, A. S., \& Hess, J. R. (2017). Understandingtests of the association of categorical variables: the Pearson chi-square test and Fisher's exact test. Transfusion, 57(4), 877-879. https://doi.org/10.1111/trf.14057

Hossain, M. A., Rashid, M. U. Bin, Khan, M. A. S., Sayeed, S., Kader, M. A., \& Hawlader, M. D. H. (2021). HealthcareWorkers' Knowledge, Attitude, and Practice Regarding Personal Protective Equipment for the Prevention of COVID-19. Journal of Multidisciplinary Healthcare, Volume 14, 229-238. https://doi.org/10.2147/JMDH.S293717

Kim, H.-Y. (2017). Statistical notes for clinical researchers: Chi-squared test and Fisher'sexact test. Restorative Dentistry \& Endodontics, 42(2), 152. https://doi.org/10.5395/rde.2017.42.2.152

Ma, H., Wang, H., Fang, L., Jiang, J., Wei, M., Liu, W., Zhao, Q., Ma, J., \& Cao, W. (2004). [A case-control study on the risk factors of severe acute respiratory syndromes among healthcare workers]. Zhonghua Liu Xing Bing XueZaZhi, 25(9), 741-744. https://doi.org/15555351

MacIntyre, C. R., Wang, Q., Rahman, B., Seale, H., Ridda, I., Gao, Z., Yang, P., Shi, W., Pang, X., Zhang, Y., Moa, A., \& Dwyer, D. E. (2014). Efficacy of face masks and respirators in preventing upper respiratory tract bacterial colonization and co-infection in hospital healthcare workers. Preventive Medicine, 62, 1-7. https://doi.org/10.1016/j.ypmed.2014.01.015

Martins, C. L., Jacondino, M. B., Antoniolli, L., Braz, D. L., Bazzan, J. S., \& Guanilo, M. E. E. (2014). Equipamentos de proteção individual na perspectiva de trabalhadores que sofreram queimaduras no trabalho. Revista de Enfermagem Da UFSM, 3. https://doi.org/10.5902/2179769211060

Mermel, L. A. (2018). Eye protection for preventing transmission of respiratory viral infections to healthcare workers. Infection Control \& Hospital Epidemiology, 39(11), 1387-1387. https://doi.org/10.1017/ice.2018.232 
Research, Society and Development, v. 10, n. 12, e26101220016, 2021

(CC BY 4.0) | ISSN 2525-3409 | DOI: http://dx.doi.org/10.33448/rsd-v10i12.20016

Ministério do Trabalho. (2010). Norma regulamentadora 6 - NR 6.

Ministério do Trabalho. (2011). Norma regulamentadora 32 - NR 32.

Offeddu, V., Yung, C.-F., Low, M. S. F., \&Tam, C. (2016). Effectiveness of masks and respirator sagainst respiratory infections in healthcare workers: A systematic review and meta-analysis. International Journal of Infectious Diseases, 53, 27. https://doi.org/10.1016/j.ijid.2016.11.074

Pereira A. S. et al. (2018). Metodologia da pesquisa científica. UFSM

Picheansathian, W., \& Chotibang, J. (2015). Glove utilization in the prevention of cross transmission: a systematic review. JBI Database of Systematic Reviews and Implementation Reports, 13(4), 188-230. https://doi.org/10.11124/jbisrir-2015-1817

Rezende, K. C. A. D., Tipple, A. F. V., Siqueira, K. M., Alves, S. B., Salgado, T. D. A., \& Pereira, M. S. (2012). Adesão à higienização das mãos e ao uso de equipamentos de proteção pessoal por profissionais de enfermagem na atenção básica em saúde. Ciência, Cuidado e Saúde, 11(2). https://doi.org/10.4025/cienccuidsaude.v11i2.15204

Silva, B. U. B. D., Sampaio, J., \& Silva, V. S. da. (2016). Riscos ocupacionais associados as condições de trabalho de enfermagem em uma unidade de terapia intensiva.

Silva, F. S., Marquini, L. L., Sabadini, O. S., \& Carletti, E. Z. B. (2018). A importância da utilização dos equipamentos de proteção individual e coletiva na prevenção de acidentes. Ambiente Acadêmico, 4(1).

Sousa, F. F. de, Sousa, I. A. de, \& Oliveira, L. M. N. de. (2019). A utilização de equipamentos de proteção individual e coletiva por profissionais de saúde: revisão integrativa. Revista de Atenção à Saúde, 16(58). https://doi.org/10.13037/ras.vol16n58.5667

Souza, S. De, Miranzi, C., Mendes, C. A., \& Iwamoto, H. H. (2010). Qualidade de vida e perfil sociodemográfico de médicos da estratégia de saúde da família. RevMed Minas Gerais, 20(2), 189-197.

Suzuki, T., Hayakawa, K., Ainai, A., Iwata-Yoshikawa, N., Sano, K., Nagata, N., Suzuki, T., Wakimoto, Y., Akiyama, Y., Miyazato, Y., Nakamura, K., Ide, S., Nomoto, H., Nakamoto, T., Ota, M., Moriyama, Y., Sugiki, Y., Saito, S., Morioka, S., \& Ohmagari, N. (2021). Effectiveness of personal protective equipment in preventing severe acute respiratory syndrome coronavirus 2 infection among healthcare workers. Journal of Infection and Chemotherapy, 27(1), 120-122. https://doi.org/10.1016/j.jiac.2020.09.006

Tomasi, E., Facchini, L. A., Piccini, R. X., Thumé, E., Silveira, D. S. da, Siqueira, F. V., Rodrigues, M. A., Paniz, V. V., \& Teixeira, V. A. (2008). Perfil sóciodemográfico e epidemiológico dos trabalhadores da atenção básica à saúde nas regiões Sul e Nordeste do Brasil. Cadernos de Saúde Pública, 24(suppl 1), s193s201. https://doi.org/10.1590/S0102-311X2008001300023

Tribunal Superior do Trabalho. (2020). EPIs desempenham papel fundamental na luta pela redução de acidentes de trabalho.

Wang, L., \& Deng, Y. (2020). The Need for Ocular Protection for Health CareWorkersDuring SARS-CoV-2 Outbreak and a Hypothesis for a Potential Personal Protective Equipment. Frontiers in Public Health, 8. https://doi.org/10.3389/fpubh.2020.599757 